\title{
Proper elimination of latent variables
}

\author{
Jan Willem Polderman* \\ Departmen of Applied Mathematics, University of Twente, P.O. Box 217,7500 AE Enschede, Netherlands
}

Received 18 September 1996 received in revised form 28 July 1997; accepted 5 August 1997

\begin{abstract}
We consider behaviors in which we distinguish two types of variables, manifest variables, the variables that are of interest to the user and latent variables, the variables that are introduced to obtain a first representation. The problem is to find a representation of the manifest behavior, that is, we want to eliminate the latent variables. If the original behavior can be represented by linear differential equations with constant coefficients, then under certain conditions the same is true for the manifest behavior. In this note we formulate and study these conditions. The results are illustrated by means of some examples. As an application we study behaviors in image representation. (c) 1997 Elsevier Science B.V.
\end{abstract}

Keywords: Elimination; Behavior; Proper i/o relation

\section{Introduction and problem statement}

In this note we study the elimination problem for behavioral representations of the form:

$R\left(\frac{\mathrm{d}}{\mathrm{d} t}\right) w=M\left(\frac{\mathrm{d}}{\mathrm{d} t}\right) \ell$,

where $R(\zeta), M(\xi)$ are polynomial matrices in $\mathbb{R}^{q \times q}[\xi]$ and $\mathbb{R}^{q \times d}[\xi]$ respectively. Throughout the paper we assume that the matrix $[R(\xi) \vdots M(\xi)]$ has full row rank (recall that a polyncmial matrix has full row rank if we can select columns from that matrix which form a square matrix of which the determinant is not the zero polynomial [3]). According to [5, 7], we can always achieve this by suitable elementary row operations.

The elimination problem consists of finding a suitable representation for Eq. (1) in terms of $w$ alone. That is, we want to eliminate $t$ from Eq. (1). The behavioral framework is the right tool to study this problem. See $[1,5,7]$ and the references therein. The

\footnotetext{
${ }^{*}$ E-mail: twpolder@math.utwente.n1.
}

problem that we are particularly interested in is, under what conditions on the matrices $R(\xi), M(\xi)$ does there exist a representation of all possible trajectories $w$ in terms of linear differential equations with constant coefficients? It will be argued that this is the case if and only if the solutions $w$ of the 'candidate representation' do not induce differentiations of the free components of $w$ (by free component we mean unrestricted by the equations, see $[5,7])$. In that case we call $\ell$ properly eliminable. We are not so much interested in obtaining the representation itself, rather we want to find necessary and sufficient conditions such that $\ell$ is properly eliminable.

The function space from which we select the solutions of Eq. (1), is the space of locally integrable functions. Since locally integrable includes non-smooth functions, we call a pair $(w, \ell) \in \mathscr{L}_{1}^{\text {loc }}\left(\mathbb{R} ; \mathbb{C}^{q}\right) \times$ $\mathscr{L}_{1}^{\text {loc }}\left(\mathbb{R} ; \mathbb{C}^{d}\right)$ a weak solution of Eq. (1), if it is a solution in the distributional sense. In the sequel we only consider weak solutions. This choice of function space is attractive from a conceptual point of view. Contrary to what our definition of weak solution suggests, it does not require knowledge of 
distribution theory and still the space is large enough to be practical (in contrast to $\mathscr{C}^{\infty}$ ).

Following $[5,7]$ we introduce the set of weak solutions of Eq. (1):

$$
\begin{aligned}
\mathfrak{B}_{\mathrm{f}}:=\left\{(w, \ell) \in \mathscr{L}_{1}^{\mathrm{loc}}\left(\mathbb{R} ; \mathbb{C}^{q}\right) \mathscr{L}_{1}^{\mathrm{loc}}\left(\mathbb{R} ; \mathbb{C}^{d}\right) \mid R(\mathrm{~d} / \mathrm{d} t) w\right. \\
=M(\mathrm{~d} / \mathrm{d} t) \ell, \text { weakly }\}
\end{aligned}
$$

Also we define the set of induced trajectories $w$ :

$$
\begin{array}{r}
\mathfrak{B}_{\mathrm{m}}:=\left\{w \in \mathscr{L}_{1}^{\mathrm{loc}}\left(\mathbb{R} ; \mathbb{C}^{q}\right) \mid \exists \ell \in \mathscr{L}_{1}^{\mathrm{loc}}\left(\mathbb{R} ; \mathbb{C}^{d}\right)\right. \\
\text { such that } \left.(w, \ell) \in \mathfrak{B}_{\mathrm{f}}\right\} .
\end{array}
$$

The set $\mathfrak{B}_{\mathrm{f}}$ is usually referred to as the full behavior, and $\mathfrak{B}_{\mathrm{m}}$ is called the manifest behavior. The full behavior contains two types of variables: the manifest variable $w$ and the latent variable $t$. Latent variables arise naturally as a by-product of 'modelling from first principles'. If we are not so much interested in a representation of the full behavior, then the question arises how to represent the manifest behavior. In particular we would like to obtain a representation in terms of linear time-invariant differential equations, if at all possible. In this note we derive necessary and sufficient conditions in terms of the polynomial matrices $R(\xi), M(\xi)$ for the existence of a matrix $R^{\prime}(\xi) \epsilon$ $\mathbb{R}^{u^{\prime} \times q}[\xi]$, such that

$\mathfrak{B}_{\mathrm{m}}=\left\{w \in \mathscr{L}_{\mathrm{l}}^{\text {loc }}\left(\mathbb{R} ; \mathbb{C}^{q}\right) \mid R^{\prime}(\mathrm{d} / \mathrm{d} t) w=0\right\}$.

It will turn out that $\mathfrak{B}_{\mathrm{m}}$ is indeed represented by $R^{\prime}(\mathrm{d} / \mathrm{d} t) w=0$ if and only if there exists a partition of $(w, \ell)$ into $\left(u, y, \ell_{1}, \ell_{2}\right)$, such that the equation $\ell_{2}=0$ defines a proper input/output relation between $u$ and $\left(y, \ell_{1}\right)$. Notice that this is not an issue if the solution space would be the space of distributions or $\mathscr{C}^{\infty}$, although we think that our results are also relevant in these cases. The paper is organized as follows. In Section 2 we explain what we mean by proper eliminability. We present a criterion for proper eliminability, this criterion is translated into a concrete test on the polynomial matrices. In Section 3 we provide some easy yet illustrative examples to show how the criterion may be used. In Section 4 we consider moving average (MA) representations of autoregressive representations (AR). This example is more subtle and illustrates the power of the notion of proper eliminability. Finally we draw some conclusions.

\section{Elimination criterion}

As a first step, we transform the representation of Eq. (1) by means of pre-multiplication of $[M(\xi) \vdots R(\xi)]$ by a suitable unimodular matrix $U(\xi) \in \mathbb{R}^{g \times y}[\xi]$ (recall that a matrix $U(\xi)$ is unimodular if its determinant is a non-zero constant [3]) into a form:

$U(\xi)[R(\xi) \vdots M(\xi)]=\left[\begin{array}{cc}R_{1}(\xi) & 0 \\ R_{2}(\xi) & M_{2}(\xi)\end{array}\right]$,

with $M_{2}(\xi)$ of full row rank. We can always find such a $U(\xi)[3]$ and according to $[5,7]$ the full behavior is also represented by

$$
\begin{aligned}
& R_{1}(\mathrm{~d} / \mathrm{d} t) w=0, \\
& R_{2}(\mathrm{~d} / \mathrm{d} t) w=M_{2}(\mathrm{~d} / \mathrm{d} t) t .
\end{aligned}
$$

It is obvious that $w \in \mathfrak{B}_{\mathrm{m}}$ implies that $R_{\mathrm{l}}(\mathrm{d} / \mathrm{d} t) w=0$, however, it is less obvious, and in fact generally not true, that $R_{1}(\mathrm{~d} / \mathrm{d} t) w=0$ implies $w \in \mathfrak{B}_{\mathrm{m}}$. Nevertheless, $R_{1}(\mathrm{~d} / \mathrm{d} t) w=0$ seems to be a good candidate representation for $\mathfrak{B}_{\mathrm{m}}$. Indeed, we show that under the conditions mentioned in the introduction, this is the case. To point out the difficulty, we first provide an example where $R_{1}(\mathrm{~d} / \mathrm{d} t) w=0$ does not represent $\mathfrak{B}_{\mathrm{m}}$.

Example 2.1. Let the full behavior be represented by

$$
\begin{aligned}
& w_{1}-w_{2}=0, \\
& (\mathrm{~d} / \mathrm{d} t) w_{2}=\ell,
\end{aligned}
$$

i.e. the matrices $R(\xi), M(\xi)$ are given by

$R(\xi)=\left[\begin{array}{cc}1 & -1 \\ 0 & \xi\end{array}\right], \quad M(\xi)=\left[\begin{array}{l}0 \\ 1\end{array}\right]$.

The manifest behavior $\mathfrak{B}_{\mathrm{m}}$ is given by

$$
\begin{aligned}
\mathfrak{B}_{\mathrm{m}}= & \left\{\left(w_{1}, w_{2}\right) \in \mathscr{L}_{1}^{\text {loc }}\left(\mathbb{R} ; \mathbb{C}^{2}\right) \mid w_{1}=w_{2},\right. \\
& \exists \ell \in \mathscr{L}_{1}^{\text {loc }}(\mathbb{R} ; \mathbb{C}) \text { such that } \\
& \left.\frac{\mathrm{d}}{\mathrm{d} t} w_{2}=\ell, \text { weakly }\right\} .
\end{aligned}
$$

Since the representation of Eq. (7) is already in the form of Eq. (5), the logical candidate representation for $\mathfrak{B}_{\mathfrak{m}}$ would be

$w_{1}-w_{2}=0$.

However, if, e.g. $w_{2}(t)=\underline{1}(t)$ (the Heaviside step), then there does not exist an $t \in \mathscr{L}_{1}^{\text {loc }}(\mathbb{R} ; \mathbb{C})$ such that $\mathrm{d} w_{2} / \mathrm{d} t=\ell$. 
The reason that Eq. (10) does not represent $\mathfrak{B}_{\mathrm{m}}$ is that $\mathrm{d} w_{2} / \mathrm{d} t=\ell$ imposes a smoothness condition on $w_{2}$. Obviously the limit of a fundamental sequence of smooth functions need not be smooth. Since the set of weak solutions of a system of differential equations is closed in the sense of $\mathscr{L}_{1}^{\text {loc }},[5$, Ch. 2], smoothness conditions cannot be expressed in terms of differential equations in $w$ alone. As a consequence $\mathfrak{B}_{\mathrm{m}}$ cannot be represented by differential equations of the type of Eq. (4).

Motivated by Example 2.1, we now study the following question: Under what conditions on $R(\xi), M(\xi)$, is the manifest behavior $\mathfrak{B}_{\mathrm{m}}$ represented by Eq. (4)?

The following definition formalizes this question.

Definition 2.2. Let $\mathfrak{B}$ be the behavior with latent variables, defined by

$R(\mathrm{~d} / \mathrm{d} t) w=M(\mathrm{~d} / \mathrm{d} t) \ell$.

We call the latent variable $\ell$ properly eliminable if there exists a polynomial matrix $R^{\prime}(\xi)$ such that the manifest behavior

$\mathfrak{B}_{\mathrm{m}}:=\{w \mid \exists \ell$ such that $(w, \ell) \in \mathfrak{B}\}$

is described by

$R^{\prime}(\mathrm{d} / \mathrm{d} t) w=0$.

It is not difficult to see that $\mathfrak{B}$ is always represented by differential equations and some additional smoothness conditions. To see this, consider Eq. (6). Since $M_{2}(\xi)$ has full row rank, we can select columns from $M_{2}(\xi)$ to form a square matrix with a constant non-zero determinant. Without loss of generality we may assure that $M_{2}(\xi)=\left[M_{21}(\xi) \vdots M_{22}(\xi)\right]$, let $M_{21}(\xi) \neq 0$. Partition $\ell$ accordingly and consider

$R_{2}(\mathrm{~d} / \mathrm{d} t) w=M_{21}(\mathrm{~d} / \mathrm{d} t) \ell_{1}$.

If $M_{21}^{-1}(\xi) R_{2}(\xi)$ is proper, then for each $w \in$ $\mathscr{L}_{1}^{\text {loc }}\left(\mathbb{R} ; \mathbb{C}^{q}\right)$ there exists $\ell_{1} \in \mathscr{L}_{1}^{\text {loc }}\left(\mathbb{R} ; \mathbb{C}^{d_{1}}\right)$ such that $R_{2}(\mathrm{~d} / \mathrm{d} t) w=M_{21}(\mathrm{~d} / \mathrm{d} t) \ell_{1}$ and in that case $\mathfrak{B}_{\mathrm{m}}$ is represented by $R_{1}(\mathrm{~d} / \mathrm{d} t) w=0$. If, however, $M_{21}^{-1}(\xi) R_{2}(\xi)$ is not proper, we cannot draw that conclusion, since there could be solutions $w$ of $R_{1}(\mathrm{~d} / \mathrm{d} t) w=0$ to which there does not correspond an $\ell_{1}$ such that $R_{2}(\mathrm{~d} / \mathrm{d} t) w=M_{21}(\mathrm{~d} / \mathrm{d} t) \ell_{1}$. To have a closer look at this phenomenon, choose minimal $k \in \mathbb{N}$ such that $\xi^{-k} M_{21}^{-1}(\xi) R_{2}(\xi)$ is proper and consider

$$
\begin{aligned}
& R_{2}(\mathrm{~d} / \mathrm{d} t) w=M_{21}(\mathrm{~d} / \mathrm{d} t)(\mathrm{d} / \mathrm{d} t)^{k} \tilde{\ell}_{1}, \\
& \ell_{1}=(\mathrm{d} / \mathrm{d} t)^{k} \tilde{\ell}_{1} .
\end{aligned}
$$

Obviously, for every $\left(w, \ell_{1}\right)$ such that Eq. (14) is satisfied, there exists $\tilde{\ell}_{1}$ such that $\left(w, \ell_{1}, \tilde{\ell}_{1}\right)$ satisfies Eq. (15). Also, by construction, for every $w \in \mathscr{L}_{1}^{\text {loc }}\left(\mathbb{R} ; \mathbb{C}^{q}\right)$ there exists $\tilde{\ell}_{1}$ such that the first of Eq. (15) is satisfied. Of course, given $\tilde{\ell}_{1}$ we can find $\ell$ such that the second of Eq. (15) is satisfied provided $\tilde{\ell}_{1}$ is sufficiently smooth. This suggests that $\mathfrak{B}_{\mathrm{m}}$ is represented by $R_{l}(\mathrm{~d} / \mathrm{d} t) w=0$ and some additional smoothness conditions. Of course, these smoothness conditions need not affect every component of $w$. The key idea of our result is that smoothness of the relevant components of $w$ could very well be guaranteed by the restrictions that $R_{1}(\mathrm{~d} / \mathrm{d} t) w=0$ imposed on $w$. As an example consider the case where $R_{1}(\xi)$ is a square matrix. In that case every weak solution of $R_{1}(\mathrm{~d} / \mathrm{d} t) w=0$ is infinitely differentiable, and the smoothness required by the second equation is guaranteed by the first.

As a preparation to our main result, we present a topological characterization of proper eliminability.

Theorem 2.3. The latent variable $\ell$ is properly eliminable if and only if $\mathfrak{B}_{\mathrm{m}}$ is closed in the sense of $\mathscr{L}_{1}^{\text {loc }}$.

Proof. The only if part follows directly from the fact that behaviors of the form $R^{\prime}(\mathrm{d} / \mathrm{d} t) w=0$ are closed in the sense of $\mathscr{L}_{1}^{\text {loc }}\left(\mathbb{R} ; \mathbb{C}^{q}\right)$. A proof of this property may be found in [5, Ch. 2].

Now suppose $\mathfrak{B}_{\mathrm{m}}$ is closed. Define $\mathfrak{B}^{\prime}$ by

$$
\mathfrak{B}^{\prime}=\left\{w \in \mathscr{L}_{1}^{\text {loc }}\left(\mathbb{R} ; \mathbb{C}^{q}\right) \mid R_{1}\left(\frac{\mathrm{d}}{\mathrm{d} t}\right) w=0\right\} .
$$

We show that $\mathfrak{B}_{\mathrm{m}}=\mathfrak{B}^{\prime}$. One inclusion is trivial: $\mathfrak{B}_{\mathrm{m}} \subset \mathfrak{B}^{\prime}$. Choose $w \in \mathfrak{B}^{\prime}$. It can be shown that $\mathfrak{B}^{\prime} \cap \mathscr{C}^{\infty}$ is dense in $\mathfrak{B}^{\prime}$, see [5, Ch. 2]. Hence we can find a sequence $w_{n} \in \mathfrak{B}^{\prime} \cap \mathscr{C}^{\infty}$ such that $\lim _{n \rightarrow \infty} w_{n}=w$ (in the sense of $\mathscr{L}_{1}^{\text {loc }}\left(\mathbb{R} ; \mathbb{C}^{q}\right)$ ). Since $w_{n} \in \mathscr{C}^{\infty}$, there exists a sequence $\ell_{n}$ such that $R_{2}(\mathrm{~d} / \mathrm{d} t) w_{n}=M_{2}(\mathrm{~d} / \mathrm{d} t) \ell_{n}$. Since by assumption $R_{1}(\mathrm{~d} / \mathrm{d} t) w_{n}=0$, we conclude that $w_{n} \in \mathfrak{B}_{\mathrm{m}}$. Since $\mathfrak{B}_{\mathrm{m}}$ is closed and since $w_{n}$ converges to $w$, we conclude that $w \in \mathfrak{B}_{\mathrm{m}}$.

Inspection of the proof of Theorem 2.3 shows that $R_{1}(\mathrm{~d} / \mathrm{d} t) w=0$ is indeed a representation of $\mathfrak{B}_{\mathrm{m}}$ if $\ell$ is properly eliminable.

Corollary 2.4. Consider the representation given by $E q$. (6) which is a representation of Eq. (2). If $\ell$ 
is properly eliminable, then $\mathfrak{B}_{\mathrm{m}}$ is represented by $R_{1}(\mathrm{~d} / \mathrm{d} t) w=0$.

The following theorem characterizes exactly when the solutions of $R_{1}(\mathrm{~d} / \mathrm{d} t) w=0$ are sufficiently smooth, so that $R_{2}(\mathrm{~d} / \mathrm{d} t) w=M(\mathrm{~d} / \mathrm{d} t) \ell$ does not impose additional restrictions on $w$.

Theorem 2.5. Consider the behavior $\mathfrak{B}$ defined by $E q$. (1). Denote by $g^{\prime}$ the (row) rank of $M(\xi)$ (as a polynomial matrix). The variable $\ell$ is properly eliminable if and only if there exist permutation matrices $P_{1} \in \mathbb{R}^{q \times q}, P_{2} \in \mathbb{R}^{d \times d}$ such that if we define

$\left[\begin{array}{l}u \\ y \\ \ell_{1} \\ \ell_{2}\end{array}\right]:=\left[\begin{array}{cc}P_{1} & 0 \\ 0 & P_{2}\end{array}\right]\left[\begin{array}{l}w \\ \ell\end{array}\right]$,

$y: \mathbb{R} \rightarrow \mathbb{R}^{y-y^{\prime}}, \quad \ell_{1}: \mathbb{R} \rightarrow \mathbb{R}^{y^{\prime}}$,

then the sub-behavior $\ell_{2}=0$ defines a proper input/output relation with $u$ as the input and $\left(y, \ell_{1}\right)$ as the output.

Remark 2.6. Theorem 2.5 in words: The variable $\ell$ is properly eliminable if and only if there exists a partition of $(w, \ell)$ into $\left(u, y, \ell_{1}, \ell_{2}\right)$ such that the equation $\ell_{2}=0$ defines a proper input/output relation between $u$ and $\left(y, \ell_{1}\right)$.

Proof of Theorem 2.5. For notational convenience and without loss of generality we assume that $P_{1}$ and $P_{2}$ are identity and that $M(\xi)$ is of the form:

$M(\xi)=\left[\begin{array}{cc}0 & 0 \\ M_{21}(\xi) & M_{22}(\xi)\end{array}\right]$,

where $\left[M_{21}(\xi) M_{22}(\xi)\right]$ has full row rank. In terms of $\left(u, y, \ell_{1}\right)$, the sub-behavior $\left\{(u, y, \ell) \in \mathfrak{B} \mid \ell_{2}=0\right\}$ is described by

$$
\begin{aligned}
& R_{11}(\mathrm{~d} / \mathrm{d} t) y+R_{12}(\mathrm{~d} / \mathrm{d} t) u=0, \\
& R_{21}(\mathrm{~d} / \mathrm{d} t) y+R_{22}(\mathrm{~d} / \mathrm{d} t) u=M_{21}(\mathrm{~d} / \mathrm{d} t) \ell_{1} .
\end{aligned}
$$

By proper $\mathrm{i} / \mathrm{o}$ relation between $u$ and $\left(y, \ell_{1}\right)$ we mean that the rational matrix:

$$
\left[\begin{array}{cc}
R_{11}(\xi) & 0 \\
R_{21}(\xi) & -M_{21}(\xi)
\end{array}\right]^{-1}\left[\begin{array}{l}
-R_{12}(\xi) \\
-R_{22}(\xi)
\end{array}\right]
$$

is well-defined and proper. Choose $(u, y)$ such that

$$
R_{11}\left(\frac{\mathrm{d}}{\mathrm{d} t}\right) y+R_{12}\left(\frac{\mathrm{d}}{\mathrm{d} t}\right) u=0 .
$$

Since Eq. (19) defines a proper $\mathrm{i} / \mathrm{o}$ relation between $u$ and $\left(y, \ell_{1}\right)$, there exist $\left(\tilde{y}_{,} \tilde{\ell}_{1}\right)$ such that

$$
\begin{aligned}
& R_{11}(\mathrm{~d} / \mathrm{d} t) \tilde{y}+R_{12}(\mathrm{~d} / \mathrm{d} t) u=0, \\
& R_{21}(\mathrm{~d} / \mathrm{d} t) \tilde{y}+R_{22}(\mathrm{~d} / \mathrm{d} t) u=M_{21}(\mathrm{~d} / \mathrm{d} t) \tilde{t}_{1} .
\end{aligned}
$$

From Eqs. (21) and (22) it follows that $R_{11}(\mathrm{~d} / \mathrm{d} t)$ $(y-\tilde{y})=0$ and since $\operatorname{det} R_{11}(\xi) \neq 0$ it follows that $y-\tilde{y} \in \mathscr{C}^{\infty}$. Moreover since $\operatorname{det} M_{21}(\zeta) \neq 0$, we can find $\bar{l}_{1}$ such that

$M_{21}\left(\frac{\mathrm{d}}{\mathrm{d} t}\right) \bar{\ell}_{1}=R_{21}\left(\frac{\mathrm{d}}{\mathrm{d} t}\right)(y-\tilde{y})$.

Define $t_{1}:=\bar{\ell}_{1}+\bar{t}_{1}$, then from Eqs. (21)-(23) we conclude that

$$
\begin{aligned}
& R_{11}(\mathrm{~d} / \mathrm{d} t) y+R_{12}(\mathrm{~d} / \mathrm{d} t) u=0, \\
& R_{21}(\mathrm{~d} / \mathrm{d} t) y+R_{22}(\mathrm{~d} / \mathrm{d} t) u=M_{21}(\mathrm{~d} / \mathrm{d} t) \ell_{1} .
\end{aligned}
$$

This shows that for every $(u, y)$ that satisfies Eq. (21) we can find $\ell_{1}$ such that Eq. (24) holds. Hence the manifest behavior is defined by

$R_{1}(\mathrm{~d} / \mathrm{d} t) w=0$,

where of course $R_{1}(\xi)=\left[R_{11}(\zeta) R_{12}(\xi)\right]$. Conversely assume that $f$ is properly eliminable. By Corollary $2.4, \mathfrak{B}_{\mathrm{m}}$ is described by $R_{\mathrm{l}}(\mathrm{d} / \mathrm{d} t) w=0$. Write $R_{1}(\mathrm{~d} / \mathrm{d} t) w=0$ in proper $\mathrm{i} / \mathrm{o}$ form (see $[5,7]$ ):

$R_{11}\left(\frac{\mathrm{d}}{\mathrm{d} t}\right) y+R_{12}\left(\frac{\mathrm{d}}{\mathrm{d} t}\right) u=0$.

The correspondingly partitioned equation for $t$ becomes

$R_{21}\left(\frac{\mathrm{d}}{\mathrm{d} t}\right) y+R_{22}\left(\frac{\mathrm{d}}{\mathrm{d} t}\right) u=M_{2}\left(\frac{\mathrm{d}}{\mathrm{d} t}\right) \ell$.

Write $M_{2}(\mathrm{~d} / \mathrm{d} t) \ell=0$ in proper i/o form:

$M_{21}\left(\frac{\mathrm{d}}{\mathrm{d} t}\right) \ell_{1}+M_{22}\left(\frac{\mathrm{d}}{\mathrm{d} t}\right) \ell_{2}=0$,

where $M_{21}^{-1}(\xi) M_{22}(\xi)$ is proper. Choose $u \in \mathscr{L}_{1}^{\text {loc }}$. By assumption we know that there exists $(y, \ell)$ such that Eqs. (26) and (27) are satisfied. Since Eq. (28) is in i/o form, we can find $\bar{l}_{1}$ such that

$M_{21}\left(\frac{\mathrm{d}}{\mathrm{d} t}\right) \bar{l}_{1}-M_{22}\left(\frac{\mathrm{d}}{\mathrm{d} t}\right) l_{2}=0$.

Combining Eqs. (27) and (28) with Eq. (29) yields

$R_{21}\left(\frac{\mathrm{d}}{\mathrm{d} t}\right) y+R_{22}\left(\frac{\mathrm{d}}{\mathrm{d} t}\right) u=M_{21}\left(\frac{\mathrm{d}}{\mathrm{d} t}\right)\left(t_{1}+\bar{t}_{1}\right)$. 
The conclusion is that for every $u$ we can find $\left(y, \tilde{\ell}_{1}, 0\right)$ such that Eqs. (26) and (27) are satisfied. This gives rise to an $\mathrm{i} / \mathrm{o}$ representation of the form of Eq. (19). Finally suppose that the corresponding rational matrix given in Eq. (20) is not proper. Then there exists a $u$ for which there does not exist $\left(y, \ell_{1}\right)$ such that Eqs. (26) and (27) are satisfied. This contradicts the assumption and the result follows.

Notice that Theorem 2.5 enables us to check whether $\mathfrak{B}_{\mathrm{m}}$ admits a representation of the form of Eq. (13), without actually computing this representation.

As a simple but useful property we mention the transitivity of proper eliminability.

Lemma 2.7. Consider the full behavior $\mathfrak{B}_{\mathrm{f}}$ represented by $R(\mathrm{~d} / \mathrm{d} t) w=M(\mathrm{~d} / \mathrm{d} t) \ell$. Let $\ell=\left[\ell_{1}, \ell_{2}\right]^{\mathrm{T}}$ be a partitioning of $\ell$. Suppose that $\ell_{2}$ is properly eliminable and that the corresponding manifest behavior is represerited by $R_{1}(\mathrm{~d} / \mathrm{d} t) w=M_{1}(\mathrm{~d} / \mathrm{d} t) \ell_{1}$. Suppose that in turn $\ell_{1}$ is properly eliminable from $R_{1}(\mathrm{~d} / \mathrm{d} t) w=M_{1}(\mathrm{~d} / \mathrm{d} t) \ell_{1}$, then $\ell$ is properly eliminable from $R(\mathrm{~d} / \mathrm{d} t) w=M(\mathrm{~d} / \mathrm{d} t) \ell$.

Proof. This follows immediately from Definition 2.2.

We are aware that the formulation of Theorem 2.5 may appear not very transparent. However, despite this observation, it enables us to formulate a criterion for proper eliminability that could in principle be programmed.

Theorem 2.8. Consider the behavior $\mathfrak{B}$ defined by Eq. (1). Denote by $g^{\prime}$ the row rank of $M(\xi)$. The latent variable $\ell$ is properly eliminable if and only if we can select $g^{\prime}$ columns from $M(\xi), g-g^{\prime}$ columns from $R(\xi)$, together forming a square matrix $P(\xi)$ such that $P^{-1} Q(\xi)$ is well-defined and proper, where the matrix $Q(\xi)$ consists of those columns of $R(\xi)$ that are not used for $P(\xi)$.

Alternatively: Consider all possible $g \times g$ square sub-matrices of $[R(\xi) M(\xi)]$. The variable $\ell$ is properly eliminable if and only if among all possible square sub-matrices of maximal determinant degree, there is one that contains exactly $g^{\prime}$ columns of $M(\xi)$.

\section{Examples}

In this section we present some examples to illustrate how to check whether or not a latent variable is properly eliminable. In Example 3.1 we show that the state in state-space representation of input/output systems is properly eliminable, in Example 3.2 we discuss proper elimination in parallel interconnections. These examples are rather straightforward and confirm the intuition.

Example 3.1. Consider the input/state/output behavior defined by

$$
\begin{aligned}
& \frac{\mathrm{d}}{\mathrm{d} t} x=A x+B u, \\
& y=C x+D u .
\end{aligned}
$$

The state $x$ is properly eliminable.

Proof. Define $R(\xi), M(\xi)$ as follows:

$R(\xi)=\left[\begin{array}{cc}B & 0 \\ -D & I\end{array}\right], \quad M(\xi)=\left[\begin{array}{c}\xi I-A \\ C\end{array}\right]$,

then Eq. (31) is equivalent to $R(\mathrm{~d} / \mathrm{d} t)[u, y]^{\mathrm{T}}=$ $M(\mathrm{~d} / \mathrm{d} t) x$. We apply Theorem 2.5 . The relation between $u$ and $(x, y)$ is a proper input/output relation. To see this define

$P(\xi):=\left[\begin{array}{cc}\xi I-A & 0 \\ -C & I\end{array}\right], \quad Q(\xi):=\left[\begin{array}{l}B \\ D\end{array}\right]$.

Obviously $P^{-1}(\xi) Q(\xi)$ is a proper rational matrix. Eq. (31) are equivalent to

$P\left(\frac{\mathrm{d}}{\mathrm{d} t}\right)\left[\begin{array}{l}x \\ y\end{array}\right]=Q\left(\frac{\mathrm{d}}{\mathrm{d} t}\right) u$.

Since $P^{-1}(\xi) Q(\xi)$ is proper, it follows that the relation between $u$ and $(x, y)$ is a proper $\mathrm{i} / \mathrm{o}$ relation and from Theorem 2.5 we conclude that $x$ is properly eliminable.

Example 3.2. Consider the input/output behaviors $\mathfrak{B}_{i}$ defined by

$$
\begin{aligned}
& P_{i}\left(\frac{\mathrm{d}}{\mathrm{d} t}\right) y_{i}=Q_{i}\left(\frac{\mathrm{d}}{\mathrm{d} t}\right) u_{i}, \\
& \quad P^{-1}(\xi) Q(\xi) \text { proper, } i=1,2 .
\end{aligned}
$$

The parallel interconnection of $\mathfrak{B}_{1}$ and $\mathfrak{B}_{2}$ is defined by Eq. (35) and the additional equations:

$u=u_{1}, \quad u_{1}=u_{2}, \quad y=y_{1}+y_{2}$.

Keeping in mind the transitivity property (Lemma 2.7), we first eliminate $u_{1}$ and $u_{2}$. It is trivial to see 
that after elimination of $u_{1}, u_{2}$ the equations become

$P_{i}\left(\frac{\mathrm{d}}{\mathrm{d} t}\right) y_{i}=Q_{i}\left(\frac{\mathrm{d}}{\mathrm{d} t}\right) u, \quad i=1,2, \quad y=y_{1}+y_{2}$.

In the form of Eq. (1):

$$
\begin{aligned}
& {\left[\begin{array}{cc}
Q_{1}(\mathrm{~d} / \mathrm{d} t) & 0 \\
Q_{2}(\mathrm{~d} / \mathrm{d} t) & 0 \\
0 & I
\end{array}\right]\left[\begin{array}{l}
u \\
y
\end{array}\right]} \\
& =\left[\begin{array}{cc}
P_{1}(\mathrm{~d} / \mathrm{d} t) & 0 \\
0 & P_{2}(\mathrm{~d} / \mathrm{d} t) \\
I_{1} & I_{2}
\end{array}\right]\left[\begin{array}{l}
y_{1} \\
y_{2}
\end{array}\right] .
\end{aligned}
$$

Obviously the right-hand side polynomial matrix of Eq. (38) does not have full row rank and hence we can apply Theorem 2.5. The relation between $u$ and $\left(y_{1}, y_{2}, y\right)$ is given by

$$
\begin{aligned}
& {\left[\begin{array}{c}
Q_{1}(\mathrm{~d} / \mathrm{d} t) \\
Q_{2}(\mathrm{~d} / \mathrm{d} t) \\
0
\end{array}\right] u} \\
& =\left[\begin{array}{ccc}
P_{1}(\mathrm{~d} / \mathrm{d} t) & 0 & 0 \\
0 & P_{2}(\mathrm{~d} / \mathrm{d} t) & 0 \\
I_{1} & I_{2} & -I
\end{array}\right]\left[\begin{array}{l}
y_{1} \\
y_{2} \\
y
\end{array}\right] .
\end{aligned}
$$

It is clear that since $P_{i}^{-1}(\xi) Q_{i}(\xi)$ is proper $(i=1,2)$, Eq. (39) defines a proper input/output relation between $u$ and $\left(y_{1}, y_{2}, y\right)$. The conclusion is that $\left(u_{1}, u_{2}, y_{1}, y_{2}\right)$ is properly eliminable.

Example 3.3. Let the full behavior be described by $\mathrm{d} w_{1} / \mathrm{d} t+w_{2}=\ell$, then it is not difficult to check that $\ell$ is not properly eliminable. If, however, we replace the right-hand side by $\mathrm{d} \ell / \mathrm{d} t+\ell$, then $\ell$ is properly eliminable.

Remark 3.4. Notice that in all of the examples we have only checked whether or not $\ell$ is properly eliminable without actually calculating the representation of the manifest behavior. For instance in Example 3.2 the representation of the manifest behavior depends on whether or not $P_{1}(\xi)$ and $P_{2}(\xi)$ are right coprime. See [1] for details.

\section{Image representations}

Consider the moving average or image representation:

$w=M\left(\frac{\mathrm{d}}{\mathrm{d} t}\right) \ell, \quad M(\xi) \in \mathbb{R}^{q \times d}[\xi]$.

In general $M(\xi)$ is not properly eliminable as the following example shows:

$M(\xi)=\left[\begin{array}{ll}1 & \xi \\ 0 & 1 \\ 0 & 0\end{array}\right]$.

It is obvious that the maximal degree determinant is obtained by selecting the second column of $M(\xi)$ and the second and the third column of $R(\xi)$, which in this case is just the identity matrix. The determinant of the resulting matrix has degree one, whereas if we would take two columns of $M(\xi)$, we would get degree zero. It follows from Theorem 2.8 that $\ell$ is not properly eliminable.

Application of Theorem 2.8 to Eq. (40) immediately yields:

Theorem 4.1. In the behavior defined by Eq. (40), $\ell$ is properly eliminable if and only if among all minors of $M(\xi)$, the maximal degree is assumed for a full size minors.

A sufficient condition on $M(\xi)$ for proper eliminability is that $M(\xi)$ is column reduced [2].

Lemma 4.2. Consider the behavior defined by Eq. (40). If $M(\xi)$ is column reduced, then $\ell$ is properly eliminable.

Proof. Since $M(\xi)$ is column reduced, there exists a $d \times d$ sub-matrix of $M(\xi)$ with proper inverse (see [2]). Hence by adding appropriate columns from the identity matrix to $M(\xi)$, we obtain a square matrix with proper inverse. Theorem 2.8 yields the statement.

Proper eliminability does not imply that $M(\xi)$ is column reduced, as the following example shows. Take

$\left[\begin{array}{ll}\xi^{3} & \xi \\ \xi & 1 \\ 0 & 0\end{array}\right]$. 
It is clear that $M(\xi)$ is not column reduced (nor row reduced). However, it follows from Theorem 2.3 that $\ell$ is properly eliminable.

In [7, Theorem V.3], it is claimed that every controllable AR representation, i.e., of the form $R(\mathrm{~d} / \mathrm{d} t) w=0$, with $R(\lambda)$ of full rank for all $\lambda \in \mathbb{C}$, admits an MA representation. In view of the preceding examples it is clear that the converse of this statement is definitely not true, there are MA representations for which $\ell$ is not properly eliminable. The proof in [7], which is actually in [6] (Proposition 4.3), is for the discrete time case for which one can argue that proper eniminability is not an issue. Notice, however, the remarks in the Conclusions. Therefore we present and prove the following result.

Theorem 4.3. Let $R_{(}(\xi) \in \mathbb{R}^{g \times q}[\xi]$ and assume that the behavior defined by $R(\mathrm{~d} / \mathrm{d} t) w=0$ is controllable, i.e., $R(\lambda)$ has full row rank for all $i \in \mathbb{C}$. There exists a matrix $M(\xi) \in \mathbb{R}^{g \times q-y}[\xi]$ such that in $w=M(\mathrm{~d} / \mathrm{d} t) \ell$, $t$ is properly eliminable and after elimination of $\ell$ we recover $R(\mathrm{~d} / \mathrm{d} t) w=0$.

Proof. Since $R(\lambda)$ has full row rank for all $\lambda \in \mathbb{C}$, there exists a matrix $R^{\prime}(\xi) \in \mathbb{R}^{q-y \times q}[\xi]$ such that:

$U(\xi):=\left[\begin{array}{c}R(\xi) \\ \cdots \\ R^{\prime}(\xi)\end{array}\right]$

is unimodular. Of course $R^{\prime}(\xi)$ is not unique, for every unimodular matrix $V(\xi) \in \mathbb{R}^{(q-g) \times(q-g)}[\xi]$, the matrix

$$
\left[\begin{array}{c}
R(\xi) \\
\cdots \\
V(\xi) R^{\prime}(\xi)
\end{array}\right]
$$

is also unimodular. Define $M(\xi)$ by

$$
M(\xi):=U(\xi)^{-1}\left[\begin{array}{cc}
! & 0 \\
0 & V^{-1}(\xi)
\end{array}\right]\left[\begin{array}{l}
0 \\
I
\end{array}\right] .
$$

Define $\tilde{U}(\xi):=U(\xi)^{-1}$. Then

$$
M(\xi)=\left[\begin{array}{c}
\tilde{U}_{12}(\xi) \\
\tilde{U}_{22}(\xi)
\end{array}\right] V^{-1}(\xi) .
$$

In general, $M(\xi)$ will not be column reduced, but of course we can choose $V(\xi)$ such that $M(\xi)$ is column reduced [2]. Hence we could have chosen $R^{\prime}(\xi)$ such that the second block column of $\tilde{U}(\xi)$ is column reduced. By Lemma 4.2, it follows that $\ell$ is properly eliminable from $w=M(\mathrm{~d} / \mathrm{d} t) \ell$. Pre-multiplication of both sides with $U(\xi)$ yields

$U(\xi)[I-M(\xi)]=\left[\begin{array}{cc}R(\xi) & 0 \\ R^{\prime}(\xi) & -I\end{array}\right]$.

By Corollary 2.4 it follows that the external behavior of $w=M(\mathrm{~d} / \mathrm{d} t) \ell$ is indeed represented by $R(\mathrm{~d} / \mathrm{d} t) w=0$.

Example 4.4. Let $R(\xi)$ be given by

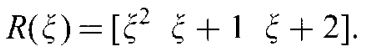

$R(\xi)$ may be completed to a unimodular matrix as follows:

$U(\breve{\zeta})=\left[\begin{array}{ccc}\xi^{2} & \xi+1 & \xi+2 \\ -\xi+1 & -1 & \xi^{2}+3 \\ \xi^{2}+\xi-1 & \xi+2 & -\xi^{2}+\xi\end{array}\right]$.

It follows that

$$
\begin{aligned}
& U^{-1}(\xi)\left[\begin{array}{l}
0 \\
I
\end{array}\right] \\
& \quad=\left[\begin{array}{cc}
-4-3 \xi-\xi^{2}-\xi^{3} & -5-4 \xi-\xi^{2}-\xi^{3} \\
-2+\xi+3 \xi^{2}+\xi^{4} & -2+\xi+4 \xi^{2}+\xi^{4} \\
1 & 1
\end{array}\right] .
\end{aligned}
$$

Calculations yield that by adding a standard basis vector to $M(\xi)$, we can obtain at most degree of the determinant equal to 2 , whereas by choosing one column from $M(\xi)$ and adding two appropriate standard basis vectors yields degree 4 . Therefore if $M(\xi)$ is defined by Eq. $(50), \ell$ is not properly eliminable. However, post-multiplication of Eq. (50) by the unimodular matrix

$$
V(\xi):=\left[\begin{array}{cc}
4+\xi^{2} & -1-4 \xi-\xi^{3} \\
-3-\xi^{2} & 1+3 \xi+\xi^{3}
\end{array}\right]
$$

yields

$$
\begin{aligned}
M(\xi):= & {\left[\begin{array}{cc}
-4-3 \xi-\xi^{2}-\xi^{3} & -5-4 \xi-\xi^{2}-\xi^{3} \\
-2+\xi+3 \xi^{2}+\xi^{4} & -2+\xi+4 \xi^{2}+\xi^{4} \\
1 & 1
\end{array}\right] } \\
& \times\left[\begin{array}{cc}
4+\xi^{2} & -1-4 \xi-\xi^{3} \\
-3-\xi^{2} & 1+3 \xi+\xi^{3}
\end{array}\right] \\
= & {\left[\begin{array}{cc}
-1 & -1 \\
-2+\xi & 2 \xi \\
1 & -\xi
\end{array}\right] . }
\end{aligned}
$$


Obviously $M(\xi)$ is column reduced so that $\ell$ is properly eliminable in $R(\mathrm{~d} / \mathrm{d} t) w=M(\mathrm{~d} / \mathrm{d} t) t$. If we write the differential equations explicitly, we find that the following two representations are externally equivalent:

$\left(\frac{\mathrm{d}}{\mathrm{d} t}\right)^{2} w_{1}+\left(\frac{\mathrm{d}}{\mathrm{d} t}+1\right) w_{2}+\left(\frac{\mathrm{d}}{\mathrm{d} t}+2\right) w_{3}=0$

and

$\left\{\begin{array}{l}w_{1}=-t_{1}-\ell_{2}, \\ w_{2}=\left(-2+\frac{\mathrm{d}}{\mathrm{d} t}\right) \ell_{1}+2 \frac{\mathrm{d}}{\mathrm{d} t} t_{2}, \\ w_{3}=\ell_{1}-\frac{\mathrm{d}}{\mathrm{d} t} \ell_{2} .\end{array}\right.$

\section{Conclusions and discussion}

We have derived a simple test for proper eliminability of latent variables. Although we only studied behaviors in continuous time, we believe that the results may also be relevant for the discrete time case. At first glance, proper elimination of latent variables seems not to be an issue in the discrete time case. For, consider Eq. (14) with the differential operator $\mathrm{d} / \mathrm{d} t$ replaced by the shift operator $\sigma((\sigma w)(k)=w(k+1))$. Since $M_{21}(\xi)$ has full row rank, there exists for each $w: \mathbb{Z} \rightarrow \mathbb{C}^{q}$ an $\ell: \mathbb{Z} \rightarrow \mathbb{C}^{d}$ such that Eq. (14) is satisfied. However, since $M_{21}(\xi)^{-1} R_{2}(\xi)$ need not be proper, the value of $t(k)$ could depend on future values of $w$. In other words $t$ may have to anticipate $w$. In applications this could be an unacceptable situation, e.g. if $\ell$ is part of a feedback loop where $\ell$ should depend causally on $w$. On the other hand if the conditions of Theorem 2.8 are satisfied, we know that not only for all $w$ that satisfy $R_{1}(\sigma) w=0$, there exists an $\ell$ such that $R_{2}(\sigma) w=M_{2}(\sigma) \ell$, but also that current values of $\ell$ do not depend on future values of $w$. An example may clarify this point. Consider the behavioral equation:

$$
\begin{aligned}
& w_{1}(k+2)+w_{1}(k+1) \\
& \quad+w_{1}(k)+w_{2}(k+1)+w_{2}(k)=0 .
\end{aligned}
$$

In polynomial notation:

$\left[\sigma^{2}+\sigma+1 \sigma+1\right]\left[\begin{array}{l}w_{1} \\ w_{2}\end{array}\right]=0$.

An image representation of Eq. (55) is

$$
\left[\begin{array}{l}
w_{1} \\
w_{2}
\end{array}\right]=\left[\begin{array}{c}
-\sigma-1 \\
\sigma^{2}+\sigma+1
\end{array}\right] t
$$

Define the unimodular matrix $U(\xi)$ by

$U(\xi):=\left[\begin{array}{cc}\xi^{2}+\xi+1 & \xi+1 \\ \xi & 1\end{array}\right]$.

After premultiplication of Eq. (56) with $U(\sigma)$ we obtain

$$
\left[\begin{array}{cc}
\sigma^{2}+\sigma+1 & \sigma+1 \\
\sigma & 1
\end{array}\right]\left[\begin{array}{l}
w_{1} \\
w_{2}
\end{array}\right]=\left[\begin{array}{l}
0 \\
1
\end{array}\right] \ell
$$

The second equation in Eq. (58) is

$w_{1}(k+1)+w_{2}(k)=\ell(k)$

and it seems that contrary to our earlier claim $\ell$ anticipates $w_{1}$. If we consider Eq. (59) as an isolated equation than this is indeed the case. However, Eq. (59) is applied only to $\left(w_{1}, w_{2}\right)$ for which the first equation in Eq. (58) is satisfied. Taking that into account, Eq. (59) becomes

$$
\begin{aligned}
\ell(k) & =w_{1}(k+1)+w_{2}(k) \\
& =w_{1}(k)-w_{1}(k-1)-w_{2}(k-1) .
\end{aligned}
$$

Eq. (60) clearly indicates that $t$ depends on current and past values of $\left(w_{1}, w_{2}\right)$ only.

In discrete time systems, proper eliminability could as well be called causal eliminability or non-anticipating eliminability.

We have illustrated the test by means of several more or less straightforward examples. A more elaborate example was provided by the application to image representations. It appeared that every $\mathrm{AR}$ representation admits a MA representation. However, not every MA representation is externally equivalent to an AR representation.

\section{Acknowledgements}

The author wishes to thank Jan C. Willems for drawing his attention to the problem and for many helpful discussions. In addition he wants to thank Margreet Kuijper for her remarks on an earlier version of this note and Paolo Rapisarda for helpful discussions. Theorem 4.1 was formulated by Robert van der Geest.

A preliminary version of the paper was presented at the IFAC World Congress in Sydney [4].

\section{References}

[1] H. Blomberg, R. Ylinen, Algebraic Theory for Multivariable Linear Systems, Academic Press, London, 1983. 
[2] T. Kailath, Linear Systems, Prentice-Hall, Englewood Cliffs, NJ, 1980.

[3] M. Newman, Integral Matrices, Academic Press, New York, 1972.

[4] J.W. Polderman, Proper elimination of latent variables, in: Proc. 12th World Congress Internat. Federation of Automat. Control, vol. 10, Sydney, Australia, 1993.
[5] J.W. Polderman, J.C. Willems, Introduction to Mathematical Systems Theory: A Behavioral Approach, Texts in Applied Mathematics 26, Springer, New York.

[6] J.C. Willems, Models for dynamics, Dynamics Reported 2 (1989) 171-269.

[7] J.C. Willems, Paradigms and puzzles in the theory of dynamical systems, IEEE Trans. Automat. Control 36 (1991) 259-294. 